relevant to relations between work factors and stress and injury occurrence is limited.

Objectives To identify the potential relations between: 1) work-related factors and stress and 2) stress and depression and injury outcomes in a high risk, understudied population.

Methods Specially designed questionnaires were disseminated to 1,200 full-time unionized janitors to collect information on their injury occurrences, personal characteristics, health history, and work-related exposures, for two sequential six-month periods. Risk ratios (RRs) and 95\% confidence intervals (CIs) were calculated using multivariable Poisson regression with robust error variances, and included bias adjustment for nonresponse and adjustment for within-person correlation using general estimating equations (GEEs).

Results 527 total observations among 390 janitors identified associations between the following exposures and high/low stress outcome (collapsed 5 point Likert scale - often/very much versus (vs) not at all/very little/sometimes): work environment factors (range $=1$, terrible/unhappy/mostly dissatisfied; 2, mixed feelings; 3, mostly satisfied/pleased delighted) how they felt about their job (1 vs 3 - RR 4.50; CI 2.38, 8.52); where they worked ( 1 vs 3 - RR 3.46; CI 2.03, 5.92); resources available for their job (1 vs 3 - RR 1.77 CI 1.14, 2.76); and job mentally or physically demanding (high/very high vs very low/low/medium demand) RR 2.49; CI 1.26, 4.93 and RR 3.74; CI 1.37, 10.25, respectively. High vs low stress exposure was associated with outcomes: diagnosed depression yes/no (RR 4.79; CI 2.22, 10.36); and risk of injury (RR 1.45; CI 1.00, 2.10).

Conclusions This analysis enabled identification of: workrelated factors associated with reported stress; the relation between stress and depression; and risk of injury among those reporting stress levels. These findings serve as a basis for future research and relevant interventions to facilitate optimal working environments.

\section{P-62 SICKNESS ABSENCE AND PSYCHOSOCIAL CONSTRAINTS AT WORK: A CROSS-SECTIONAL STUDY AT A UNIVERSITY HOSPITAL IN TUNISIA}

${ }^{1}$ Aouatef Mahfoudh, Nesrine Mars, Noura Bel Haj, Ines Rassas, Amira Omrane, Taoufik Khalfallah. 'University of Monastir, Tunisia

\subsection{6/OEM-2021-EPI.182}

Background The relationship between work and health is complex and bidirectional. It has individual and collective aspects resulting from working conditions and interpersonal relationships. Absenteeism, a complex phenomenon, represents for some workers the expression of health and work relationship. It is considered as an indicator of a person's physical and mental health status. Aims: To identify determinants of sickness absence among nurses in a Tunisian university hospital.

Methods A cross-sectional study carried out in a Tunisian University Hospital. We included medical absences declared to the employer by nurses during one year. The study was conducted in two successive phases: data collection of absence from the hospital direction (human resources), then a standardized self-administered questionnaire was distributed to the target population. This questionnaire included a socioprofessional survey and a questionnaire on Psychological and
Organizational Constraints (POC), using a version applicable to nurses (IDE).

Results The global absence rate was 6.58. Absenteeism was statistically associated with age $(p=0.035)$, marital status ( $p$ $=0.005)$ and the presence of medical and surgical history $(\mathrm{p}$ $\left.<10^{-3}\right)$. The number of absence days was statistically higher among nurses with a pathological professional history $(\mathrm{p}=$ 0.019 ) and among those with an occupational tenure higher than 15 years $(\mathrm{p}=0.034)$. For the psycho-organizational constraints studied, the period of absence was statistically associated with the organization that did not allow communication $(p=0.001)$, the lack of support from the health manager $(p$ $=0.001)$ and a shortage of staff $\left(\mathrm{p}<10^{-3}\right)$. The multivariate regression analysis showed as determinants of absenteeism: medical history $\left(\mathrm{p}<10^{-3} ; 95 \% \mathrm{CI}=[2.21 ; 8.18]\right)$, surgical history $(p=0.001 ; 95 \% \mathrm{CI}=[1.71 ; 9.12])$ and the lack of support from the health manager $(\mathrm{p}=0.001 ; 95 \%$ CI $=$ $[1.58 ; 6.34])$.

Conclusion This study allowed us to identify some determinants of absenteeism among nurses.

\section{P-63 SOCIO-ECONOMIC ISSUES OF OCCUPATIONAL MUSCULOSKELETAL DISORDERS}

${ }^{1}$ Aouatef Mahfoudh, Olfa Jlassi, Amira Omrane, Noura Bel Haj, Asma Kheder, Ines Rassas, Taoufik Khalfallah. 'University of Monastir, Tunisia

\subsection{6/OEM-2021-EPI.183}

Aims To assess determinants of absenteeism among employees with occupational musculoskeletal disorders (MSDs).

Methods A descriptive cross-sectional study carried out at the Department of Occupational Medicine in the University Hospital of Tunisia. The survey took place during the year 2019 and was conducted using a pre-established questionnaire, relating to the description of socio-demographic data and the assessment of work capacity by the Work Ability Index questionnaire 'WAI'. The study of absenteeism linked to MSDs was carried out using the Work Performance Questionnaire 'WPQ'.

Result A total of 63 participants, all females, mean age $41 \pm$ 8 years, had consulted for a musculoskeletal problem. In this series, $58 \%$ of these women had a low level of education (primary). They were married in $77 \%$ of cases with more than two children in charge in $61 \%$ of cases. The average professional length was $20 \pm 7$ years. Sectors of activity were clothing (92\%), food processing (4\%), and the automotive wiring industry (4\%). The capacity for work perceived by all the operators was low in $85 \%$ of cases and average in $15 \%$ of the cases. The mean relative absenteeism of the study population was $31 \%$. Absenteeism was significantly correlated: with age $(\mathrm{p}=0.03)$, with the work of the spouse $(\mathrm{p}=$ 0.006), with the distance between the place of work and the residence $(p=0.015)$, with the sector $(p=0.001)$ and professional seniority $(p=0.032)$. In this work, the explanatory model of medical absenteeism linked to work-related MSDs was statically linked to professional length of service ( $p$ $=0.02 ;$ OR $=2.3, \mathrm{IC}=[1.3-4.5])$.

Conclusion Musculoskeletal disorders have various repercussions in terms of suffering and interruption of professional careers of employees, but also high economic costs. As a result, a comprehensive and integrated prevention approach must be put in place. 